\title{
Yard Work
}

National Cancer Institute

\section{Source}

National Cancer Institute. Yard Work. NCI Thesaurus. Code C94748.

The act of tending a yard as a chore or hobby. 